\title{
Functional disturbance in hiatal hernia in infants and children ${ }^{1}$
}

\author{
A.R. CHRISPIN AND G. W. FRIEDLAND \\ From the Hospital for Sick Children, Great Ormond Street, London, W.C.I
}

\begin{abstract}
Retrograde flow from the stomach into the oesophagus in infants and children is prevented by the mucosal choke and muscle cuff of the normally located oesophageal vestibule (Chrispin, Friedland, and Wright, 1967). When a hiatal hernia is present the cardinal functional abnormality is the retrograde flow of gastric content into the oesophagus. An analysis of the factors permitting such flow in these patients is presented in this paper. The important anatomical features are (1) the location of the oesophageal vestibule in the thorax, (2) the phreno-oesophageal membrane with its attachment to both the diaphragm and the vestibule, and (3) the size of the hiatus in the diaphragm. When the oesophageal vestibule lies entirely in the thorax its whole length lies in a zone of subatmospheric pressure. The sharp falls of intrathoracic pressure associated with crying are related to opening of the vestibule. Reduction in tension in the muscle cuff following a dry swallow may also result in vestibular opening with retrograde flow. Inefficient secondary stripping waves may fail to empty the refluxed gastric content from the oesophagus, permitting peptic oesophagitis to develop. The increased tendency to open and the increased difficulty in closing the oesophageal vestibule, because of tension in the abnormally located phreno-oesophageal membrane, are considered. The paradox of free retrograde flow with a small hernia and small retrograde flow with a large hernia is resolved. Basic radiological criteria for establishing the diagnosis of hiatal hernia in this age group are given. The objectives of surgery and the means by which they may be achieved are discussed.
\end{abstract}

In infants and children the principal symptoms of hiatal hernia are related to retrograde flow from the stomach into the oesophagus. An analysis of the factors which permit such gastro-oesophageal reflux is presented in this paper. Initially, brief reference is made to the way in which reflux is prevented in the normal child, for this facilitates an understanding of the factors leading to reflux in hiatal hernia. The radiological criteria for the diagnosis of hiatal hernia in this age group are enumerated. Some aspects of the surgical correction of the abnormality are noted.

\section{NORMAL STRUCTURE OF THE OESOPHAGEAL VESTIBULE}

The oesophageal vestibule lies at the lower end of the oesophagus immediately proximal to the stomach. It is located partially in the thorax,

${ }^{1}$ Based on a paper presented at the Symposium on the Structure Based on a paper presented at the Symposium on the Structure
and Function of the Gastro-oesophageal Junction at the University of Cambridge on 30 September 1966 partially in the oesophageal hiatus in the diaphragm, and partially in the abdomen. Key anatomical features include an inner lining of $\underset{x}{\otimes}$ mucosa enclosed by a cuff of muscle, and this is joined to the diaphragm by the phrenooesophageal membrane. When the vestibule is closed the mucosa lies in longitudinal folds within the muscle cuff. As the vestibule opens these folds 윽 are effaced and are no longer perceptible when $D$ the vestibule is fully open (Fig. 1).

\section{FUNCTIONS OF THE OESOPHAGEAL VESTIBULE}

Manometric studies in infants have shown a疋 segment of terminal oesophagus with a highero pressure than that in the stomach or in the oesoghagus above (Carré and Astley, 1958). The $\stackrel{?}{+}$ pressure in this segment falls after the act of 0 deglutition (Gryboski, Thayer, and Spiro, 1963).

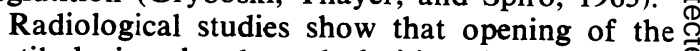
vestibule is related to deglutition (Chrispin and $\stackrel{\mathbb{Q}}{\varrho}$ Friedland, 1966). When it opens flow through it 


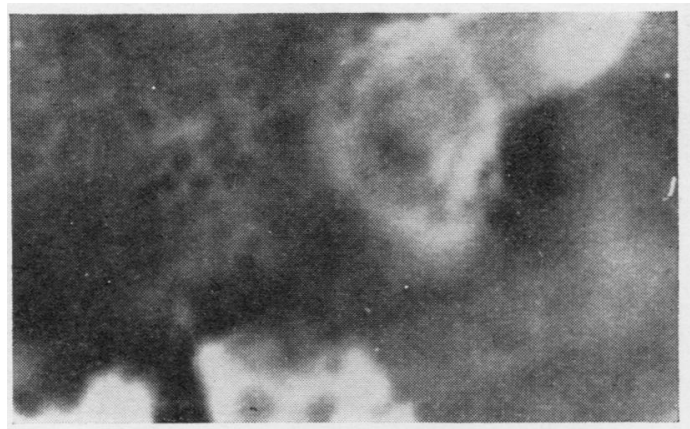

(a)

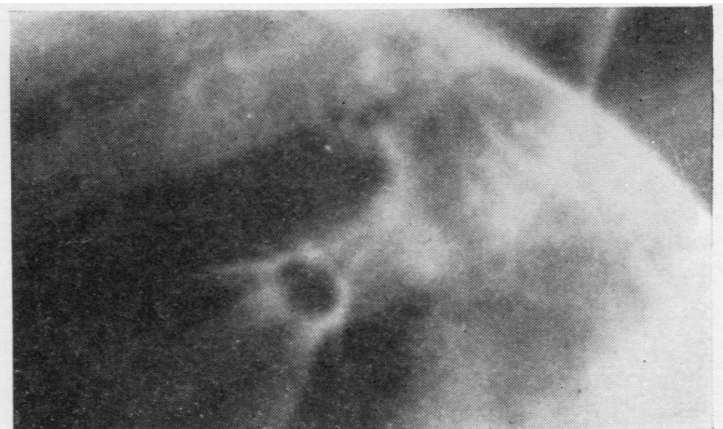

(b)

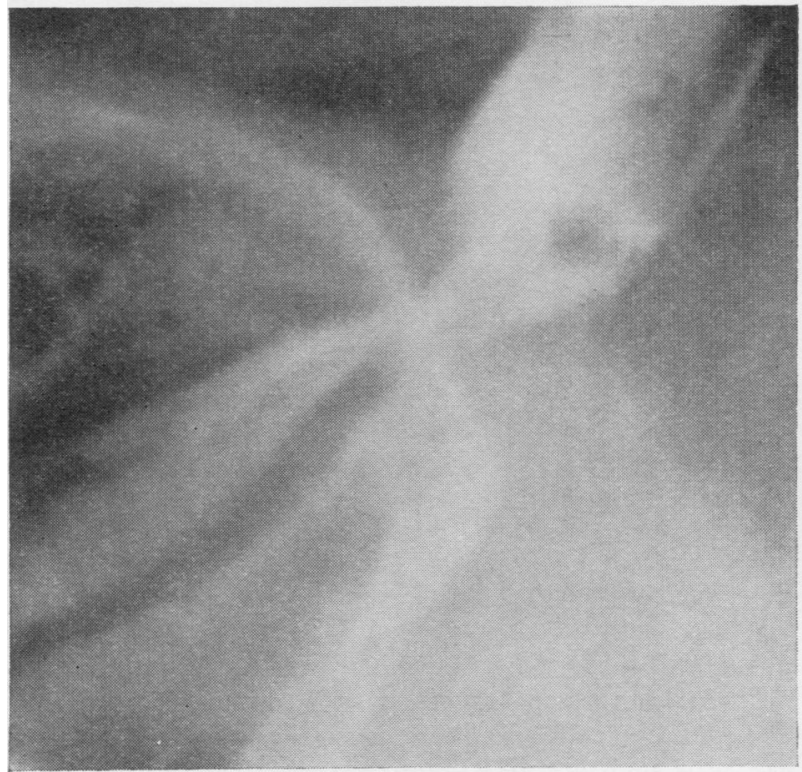

Fig. 1. (a) Vestibule fully open, patient semiprone swallowing air: no mucosal folds are visible (age 7 months). (b) Same patient as in (a): vestibule is partially open and small longitudinal folds are visible. (c) Second patient swallowing barium: vestibule is partially open with deep mucosal folds indenting column of barium (age 3 years). (d) Third patient: vestibule closed, forming an 'empty segment'. Note that when the vestibule closes gastric mucosal folds radiate from the point of closure, the so-called 'mucosal rosette' (age 6 weeks).

(c)

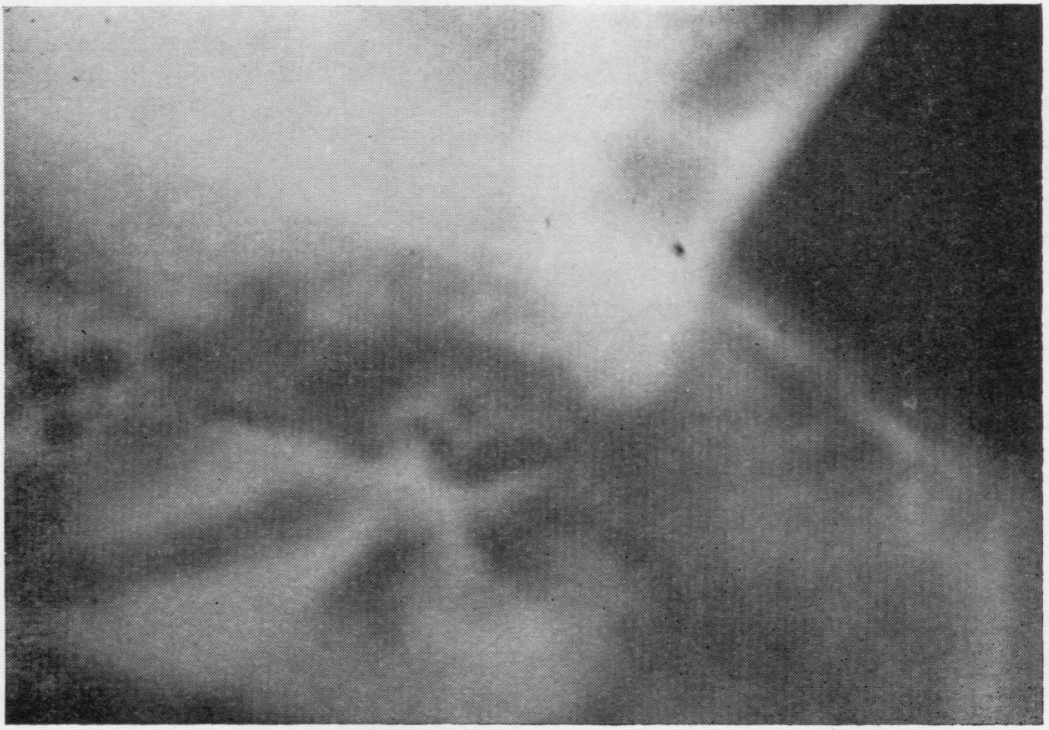

(d) 
takes place. This relationship is independent of the nature of the gullet above and is not dependent on an oesophageal stripping wave. It has been deduced that folding of the mucosa as the vestibule closes impedes flow through it (Chrispin, Friedland, and Wright, 1967). In effect, the longituninal folds of the closed vestibule provide a highly efficient choke, reducing flow. The muscle cuff permits opening and closing of the vestibule. Rises in exogastric intra-abdominal pressure are transmitted equally to the vestibule and to the stomach and hence the muscle cuff need maintain only a slight tension to keep the mucosal choke closed and prevent retrograde flow from the stomach into the oesophagus. In the patient with a normally located vestibule, the distribution of the phreno-oesophageal membrane makes it likely that only a minimal opening tendency, if there is indeed any opening tendency, can result from the thoraco-abdominal pressure difference acting across it.

\section{FACTORS INFLUENCING FLOW WHEN A HIATAL HERNIA IS PRESENT}

The para-oesophageal type of hiatal hernia is uncommon in infants and young children. Sliding types of hiatal hernia predominate and, in these, three principal features are important:

1. The vestibule is located in the thorax.

2. The phreno-oesophageal membrane passes from the under surface of the diaphragm through the oesophageal hiatus to be inserted into the lower oesophagus and vestibule which now lie entirely in the thorax.

3. When the oesophageal hiatus is extremely large the peritoneal cavity may be extended up through the hiatus into the thorax, and within this supradiaphragmatic extension of the peritoneal cavity may lie the stomach, omentum, and intestine. This is a relatively rare condition and it is likely that the ligamentous attachment between the vestibule and the diaphragm is virtually nonexistent. More commonly, the hiatus, although it may be large, is not associated with an upward extension of the peritoneal cavity.

Retrograde flow from the stomach into the oesophagus may be impeded at the diaphragmatic hiatus and the oesophageal vestibule.

DIAPHRAGMATIC HIATUS The folds of gastric mucosa as they pass through the hiatus may effectively close the lumen by a mucosal choke not unlike that seen in a normal vestibule (Figs 2 and

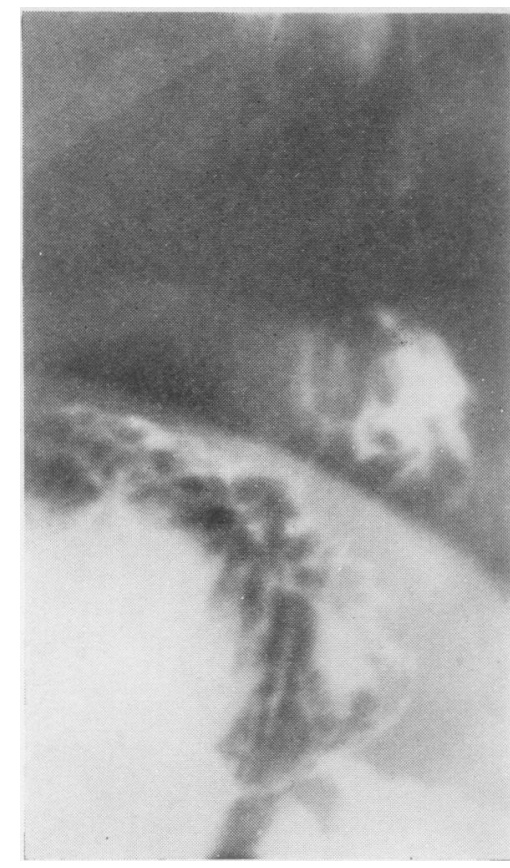

FIG. 2. Infant with hiatal hernia: gastric mucosal folds pass through the hiatus, producing a closed mucosal choke. Note that gastric mucosal folds in the infradiaphragmatic loculus radiate from the line of closure; the similarity to Fig. 1d is striking (age 4 weeks).

3). However, there is no associated intrinsic muscle cuff and there is no ligamentous support for such a choke. The choke is unstable and may open readily when the infant cries, permitting free ${ }_{-}^{\times}$ flow from the loculus of stomach below the 3 . diaphragm into the loculus above the diaphragm: (Figs 3 and 4). During crying a considerable 3 difference in pressure between the thorax and $\circ$ abdomen develops (D. G. Cottom, personal communication). Donald (1954) has drawn attentiono to the considerable power of the infant thorax, which can generate a negative intrathoracic $N$ pressure of some 60 to $80 \mathrm{~cm} . \mathrm{H}_{2} \mathrm{O}$ with the firstin breath after delivery. Free flow through the N mucosal choke in the diaphragm can be seen most ${ }_{\sigma}^{\omega}$ easily during that phase of crying in which inspiration against partially closed vocal cords? takes place.

The distinction between non-communicating and communicating hiatal herniae is largely mean- 0 ingless in infants and children. Whatever the $\overrightarrow{\mathbb{D}}$ situation may be in adult life, it is unlikely that $a \frac{\rho}{\mathbb{D}}$ flutter valve mechanism (Edwards, 1962) can be $\varrho$ invoked to explain opening of the mucosal choke 


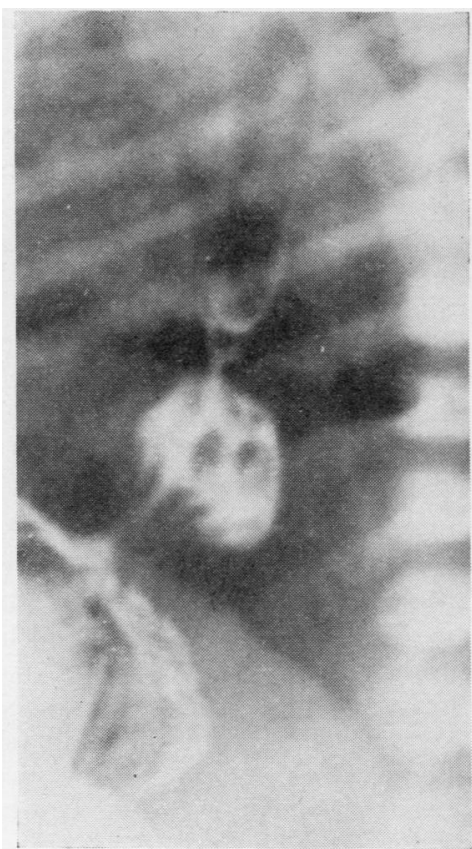

(a)

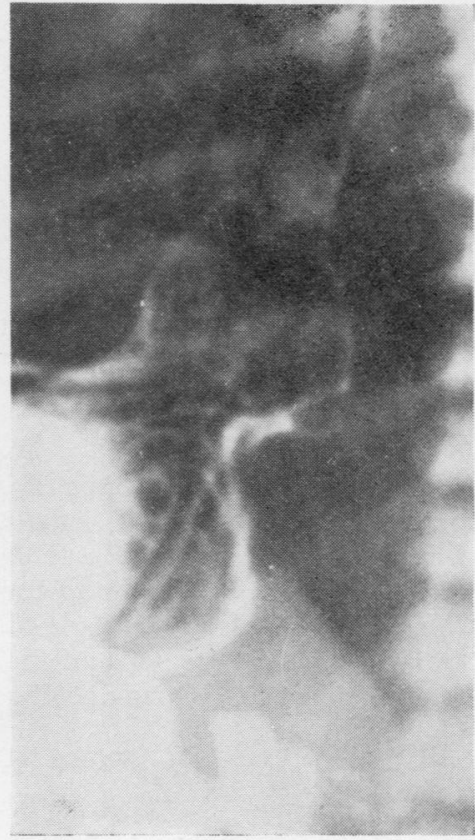

(b)

FIG. 3. Infant with hiatal hernia (age 5 days): (a) Closed gastric mucosal choke at level of diaphragm, closed vestibule above supradiaphragmatic loculus; (b) on crying gastric mucosal choke at diaphragm opens and oesophageal vestibule opens.

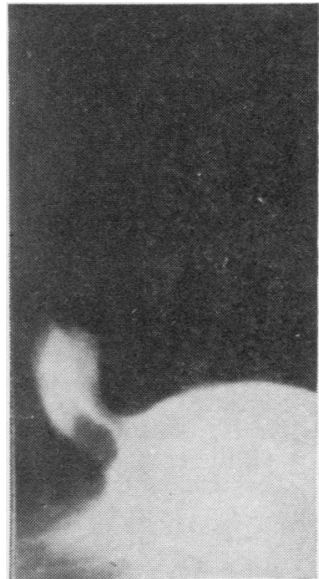

(a)

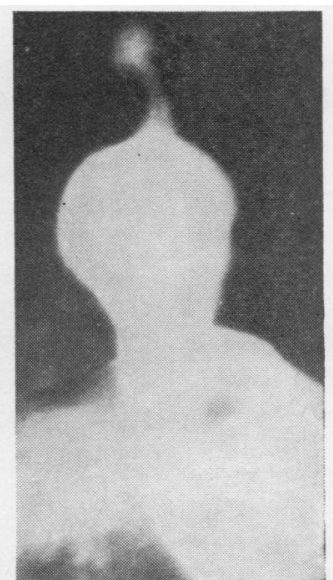

(b)

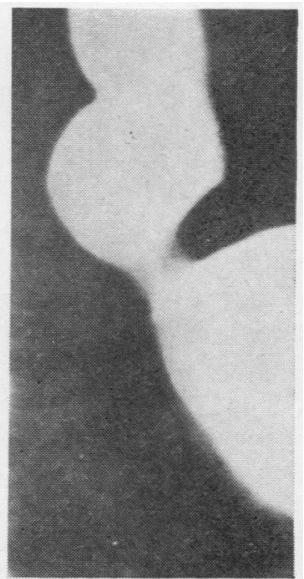

(c)

FIG. 4. Infant with hiatal hernia. Series during inspiratory cry against partially closed vocal cords (age 6 weeks): (a) mucosal choke at diaphragm beginning to open with retrograde flow through it; (b) mucosal choke at diaphragm fully open; vestibule starting to open with flow through it into oesophagus beginning; (c) vestibule fully open with considerable retrograde flow into oesophagus. Gastric mucosal choke at hiatus is reappearing. 
during crying. The increase in pressure differential between the thorax and abdomen is associated with opening: if a flutter valve mechanism were operating, then one would expect the mucosal choke to be more firmly closed.

Infants and children spend much of their time in the recumbent position, and this facilitates the retrograde flow of liquids from the stomach.

\section{FACTORS INFLUENCING OPENING AND CLOSING OF THE VESTIBULE IN THE THORAX}

Thoraco-abdominal pressure difference In patients with hiatal hernia, the vestibule lies in the thorax and the vestibule throughout its length lies in an area of relatively lower pressure than when normally located. The pressure gradient across the wall, from the lumen of the vestibule to that in the surrounding thorax, is greater and the vestibule is continually subject to greater opening forces.

Phreno-oesophageal membrane When the stomach lies entirely within the abdomen there is no pressure differential across its walls and therefore there is no tension in the wall. However, when the stomach lies in the thorax and there is communication between supra and infra diaphragmatic loculi, there is a pressure differential across the walls of the stomach and in the phrenooesophageal membrane. At times the pressure differential may be high, e.g., during crying. It is unlikely that the stomach wall itself is directly associated with an appreciable opening force at the vestibule, for it is distensible and it is free to move within certain limits (Fig. 5). However, the phreno-oesophageal membrane is fixed to the diaphragm and to the vestibule. When the attachment of this membrane to the vestibule lies entirely in the thorax then the thoraco-abdominal pressure difference creates tension in the phrenooesophageal membrane and this tends to open the vestibule. The strength of this opening tendency will be dependent upon the size of the hiatus in the diaphragm, the strength of the phrenooesophageal membrane, the calibre of the vestibule, and the size of the thoraco-abdominal pressure difference. The greater the size of the hiatus the greater the tension in the membrane; the greater the strength of the phreno-oesophageal membrane the greater will be the opening force at the vestibule. These observations are consistent with the finding that small herniae, presumably with a strong phreno-oesophageal membrane, are associated with free retrograde flow and prominent symptoms of gastro-oesophageal reflux (Fig. 6). In larger herniae, presumably with a weak or absent phreno-oesophageal membrane, reflux sympto- $\frac{\bar{\omega}}{D}$ matology is frequently much less troublesome and $\stackrel{\nabla}{\varnothing}$ retrograde flow is less evident radiologically (Fig. \& 5). It can also be appreciated that, given a strong phreno-oesophageal membrane, increased tension. is required from the muscle cuff to close the $\overrightarrow{\vec{\omega}}$ vestibule.

Dillard (1964) pointed out the possible impor- $\overrightarrow{\vec{x}}$ tance of tension in the phreno-oesophageal i membrane as an opening force at the oesophageal o vestibule. The evidence given above confirms this view.

Deglutition response Following deglutition, the $\overrightarrow{-}$ intraluminal pressure in the vestibule falls. At $\mathbb{D}$ fluoroscopy in infants with hiatal hernia it can be $\underset{\mathbb{D}}{\stackrel{O}{(}}$ seen that the swallowing of a small amount of $\frac{\mathbb{D}}{3}$ contrast medium can be associated with free $\underset{\mathbb{D}}{ }$ retrograde flow from the stomach through the vestibule. Even a 'dry swallow' may be sufficient $\mathscr{\odot}$ to result in opening of the vestibule when it is. located in the thorax. In a child with an upper respiratory tract infection, repeated swallowing of nasal secretions may be associated with repeated opening of the vestibule. This can explain the clinical observation that symptoms in infants and $\stackrel{\odot}{\circ}$ children with hiatal hernia are frequently $\vec{\overrightarrow{ }}$ severely exacerbated by an upper respiratory tract 3 infection.

Oesophageal stripping wave When there is a hiatal hernia and free reflux, then oesophageal contractility is often poor. Distension of the oesophagus by retrograde flow of gastric content $\frac{5}{3}$ may not initiate a secondary stripping wave. The oesophagus fails to empty its content into the stomach and the result of this is that the refluxed gastric content remains in contact with the oesophageal mucosa for a prolonged period.

Oesophagitis Erosions and inflammatory changes $\stackrel{N}{\circ}$ must increase the rigidity of the mucosa and may 0 lead to submucous fibrosis and more permanent $\mathrm{W}$ rigidity. These factors could reduce the efficiency 0 of the mucosal choke. It is conceivable that such long-term changes permit reflux even when the hernia has been reduced.

THE MUCOSAL ROSETTE AND THE OESOPHAGO- $\frac{0}{\stackrel{0}{0}}$ GASTRIC ANGLE The mucosal 'rosette' (Fig. 1d) seen in the normal patient has a parallel in the $\stackrel{\mathbb{Q}}{\varrho}$ 'rosette' seen in hiatal hernia when the gastric으 


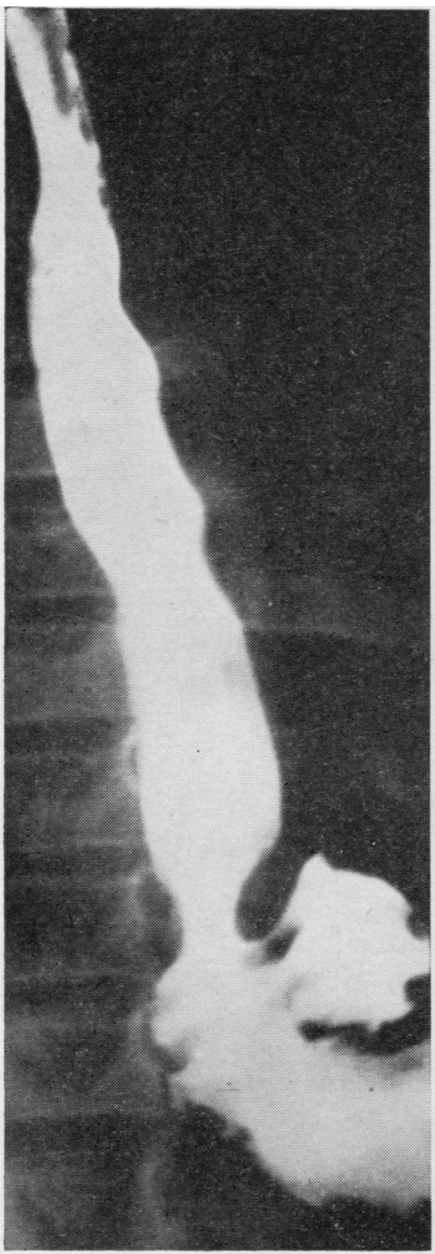

(a)

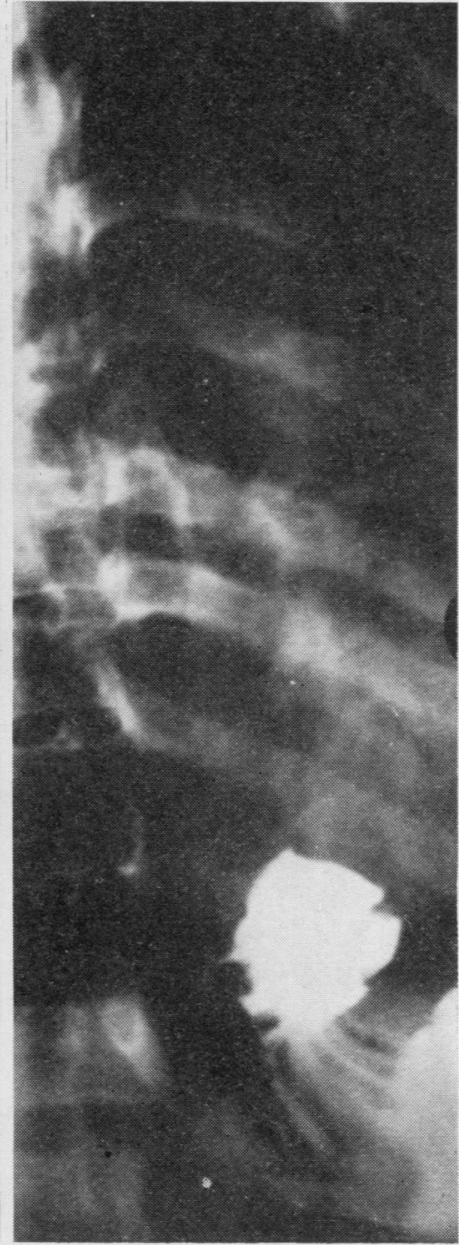

(b)

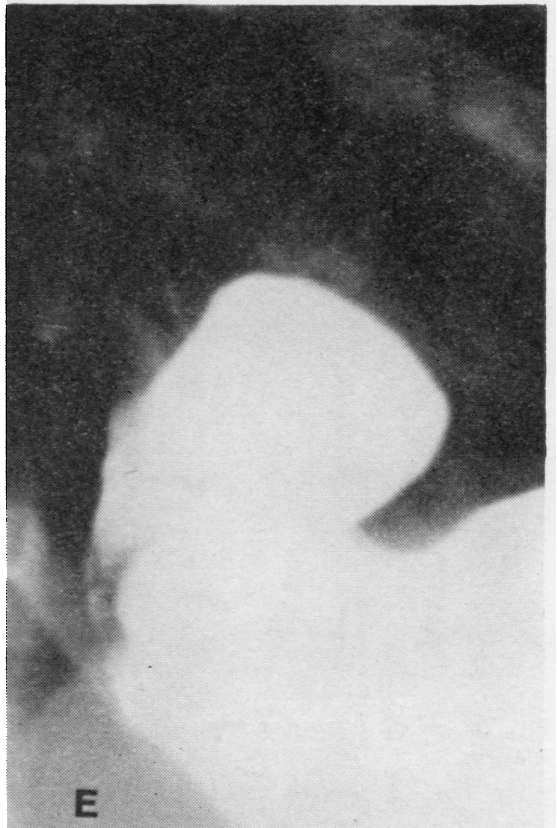

(c)

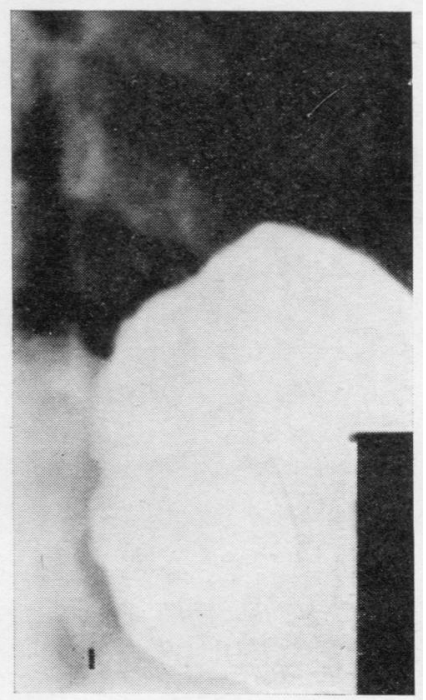

(d)

FIG. 5 e and f overleaf. 


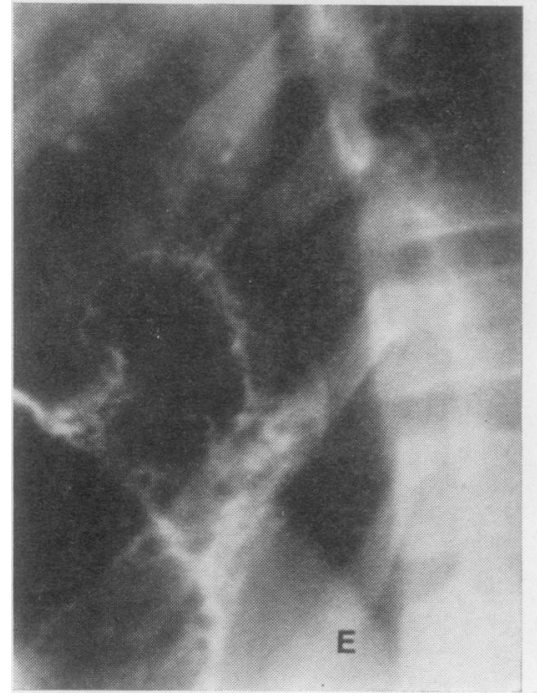

FIG. 5e. For legend see p. 427.

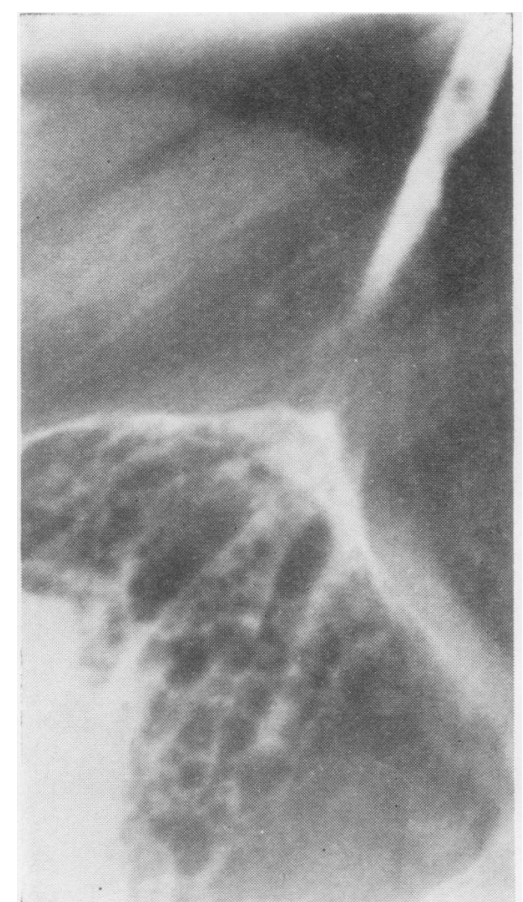

FIG. 6. Child aged 5 years. Long history of reflux symptomatology, with anaemia ( $\mathrm{Hb} 6.4 \mathrm{~g} . / 100 \mathrm{ml}$.), haematemesis, melaena, and severe nocturnal epigastric pain. Sister died elsewhere aged 2 years from oesophageal stricture. Brother aged 14 has hiatal hernia. Patient has oesophagitis on oesophagoscopy and radiologically. Minimal hiatal hernia. Free reflux radiologically and at oesophagoscopy.

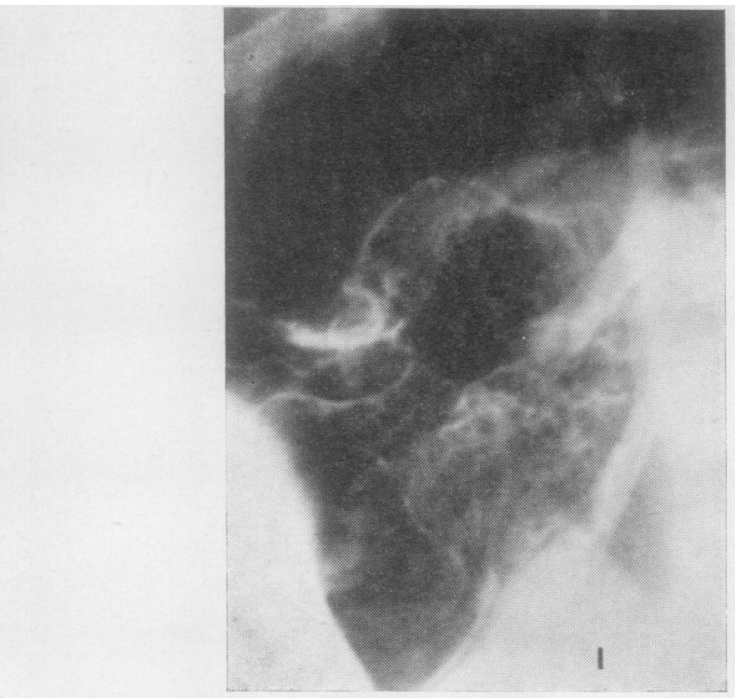

FIG. 5f. For legend see p. 427.

mucosal choke is closed (Fig. 2). It appears that the radiating folds of gastric mucosa are in each instance a result of closing of the mucosal choke. It is difficult to see that this rosette is in itself a reflux-preventing factor. Many infants and children have no perceptible acute angle of entry of the oesophagus into the stomach and no reflux symptomatology is present. That the creation of an acute angle of entry is an essential of a hiatal hernia repair is refuted by findings such as those shown in Figure 7.

\section{RADIOLOGICAL CRITERIA IN THE DIAGNOSIS OF HIATAL HERNIA}

The radiological examination of the oesophagus is carried out with the patient lying in the supine and prone left posterior oblique positions. Infants are given warmed saccharin-sweetened barium suspension to suck from a feeding bottle; older children readily suck flavoured barium suspension through a straw.

The cardinal diagnostic point is to demonstrate that for at least some of the time the vestibule lies completely above the diaphragm and in the thorax. When fully open and filled with barium the vestibule may be indistinguishable from the oesophagus above and the intrathoracic sleeve of stomach below. Its intrathoracic location can be defined as it closes, and this is better seen if the oesophagus above contains some air or barium. Vestibular opening may be observed after a dry swallow. 


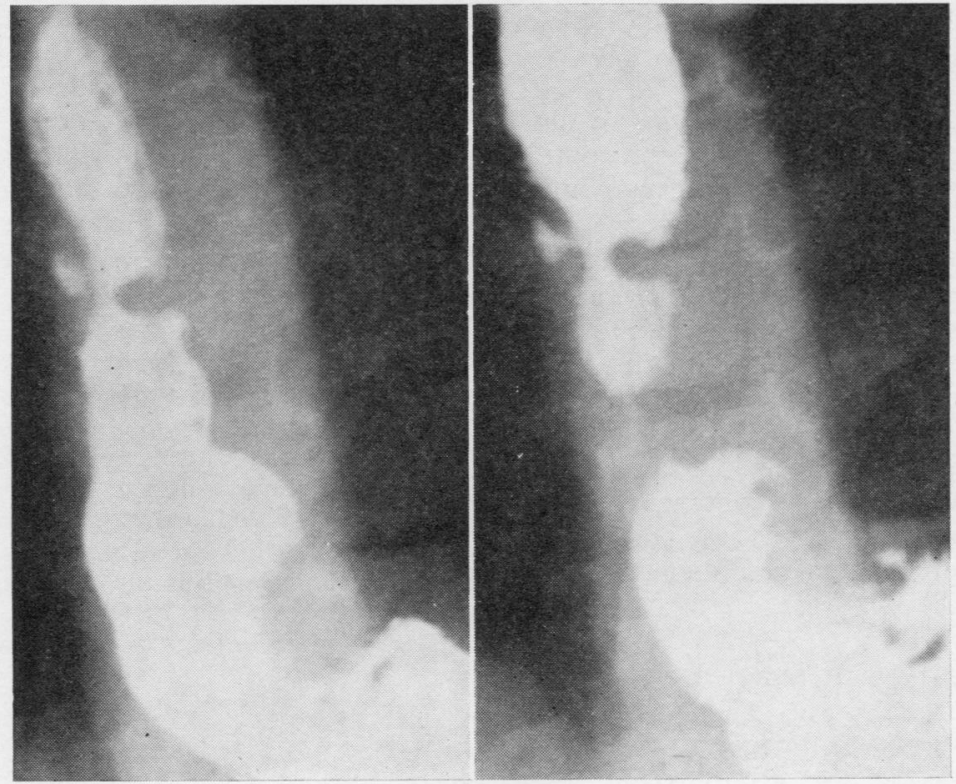

(a)

(b)

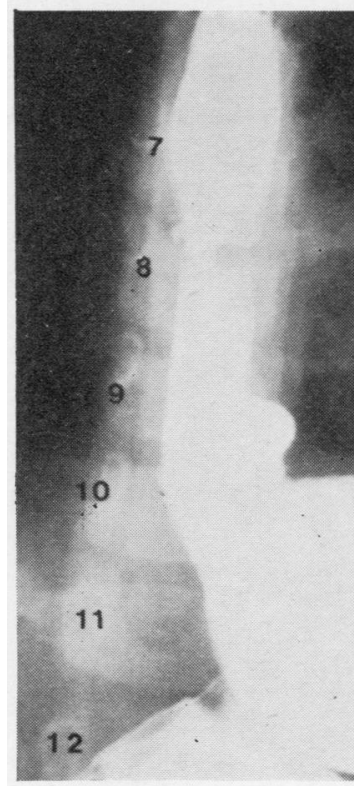

(c)

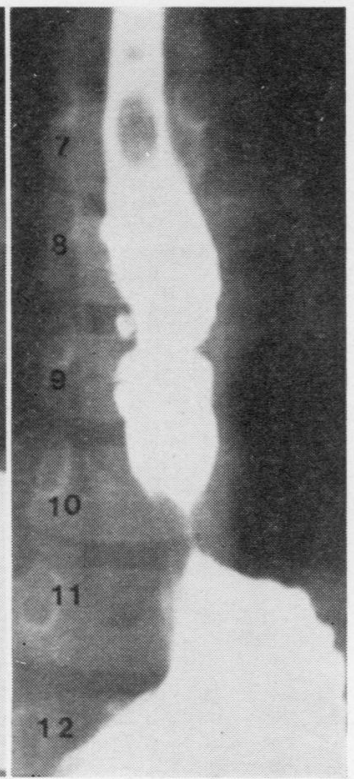

(d)

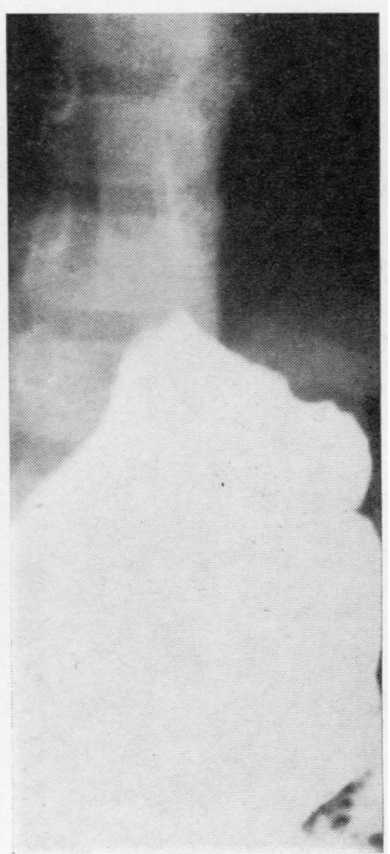

(e)

FIG. 7. Child aged 6 years: (a) pre-operatively. Oesophageal stricture and 'pocket' in oesophagus due to oesophagitis. Hiatal hernia with vestibule open. Free reflux. (b) As in (a) with vęstibule closed. (c) Pre-operative film with identical projection to that on post-operative film $(d)$. (d) Post-operatively. The vestibule can be seen to be closing at and below the level of the diaphragm (compare with (b)). (e) Post-operative film. Shows no reflux and this has been achieved without creating an oesophago-gastric angle. 
The sequence in Figs 3 and 4 shows that the effect of crying and similar changes may be associated with inspiration.

Less direct points indicating that the vestibule lies in the thorax are (1) the arrest of contrast medium above a closed vestibule with the point of arrest lying too high in the thorax; (2) the presence of numerous thick, coarse gastric folds in the hiatus when the gastric mucosal choke is closed in contrast with the finer, less numerous (commonly two to four) folds in the empty oesophagus above; and (3) the presence of many folds throughout the length of the oesophagus, suggesting that the oesophagus is accustomed to distension by reflux of gastric content.

These points do not cover the findings in stricture, oesophagitis, mega oesophagus, etc.

\section{OBJECTIVES OF SURGERY}

The functional objective of conservative surgery in hiatal hernia in this age group is to prevent retrograde flow from the stomach into the oesophagus. To achieve this it seems that the surgical technique should be directed towards producing an intra-abdominal location of at least part of the oesophageal vestibule within a hiatus of normal size. It seems to be important to do this (a) without putting stitches in the muscle cuff of the oesophageal vestibule, for these could interfere with maintenance of the slight tension needed to close the mucosal choke; $(b)$ without producing a persisting mucosal choke when the vestibule opens to its maximum : and (c) avoiding stitches in the phreno-oesophageal membrane which might increase tension in it and result in a tendency to vestibular opening.

Following operation, reflux symptomatology can ameliorate even though the hernia persists. This may be related to reduced tension in the phreno-oesophageal membrane and, less commonly, 'choking' of the herniated stomach at a narrowed hiatus.

We are indebted to our clinical colleagues, $\mathrm{Mr}$. D. J. Waterston and Professor A. W. Wilkinson, who have referred patients and have helped and encouraged the preparation of this paper. We must thank Dr. D. E. Wright, of Imperial College of Science and Technology, London, for his expert help in the field of hydraulics and for a mathematical analysis which supports and parallels the observations in this paper. We are very grateful to Dr. D. G. Cottom for details of pressure recordings in crying infants.

\section{REFERENCES}

Carré, I. J., and Astley, R. (1958). The gastro-oesophageal junction in infancy. Thorax, 13, 159.

Chrispin, A. R., and Friedland, G. W. (1966). A radiological study of the neural control of oesophageal vestibular function. Ibid.. $21,422$.

- and Wright, D. E. (1967). Some functional characteristics of the oesophageal vestibule in infants and children. Ibid., 22, 188.

Dillard, D. H. (1964). Mechanism of sphincteric failure in sliding $\frac{O}{\mathbb{}}$ hiatal hernia. In Hernia, ed. Nyhus, L. M., and Harkins, H. N. O p. 440. Pitman Medical Publishing Company, London, and $\overrightarrow{\vec{\theta}}$ Lippincott, Philadelphia.

Donald, I. (1954). Atelectasis neonatorum. J. Obstet. Gynaec. Brit. Emp., 61, 725.

Edwards, D. A. W. (1962). The 'raspberry' or 'flutter' valve in the anti-reflux mechanism. In Surgical Physiology of the Gastrointestinal Tract: Proceedings of a Symposium, Royal College of intestinal Tract: Proceedings of a Symposium, Royal Coll.
Surgeons of Edinburgh, 1962, ed. Smith, A. N., pp. 24-28.

Gryboski, J. D., Thayer, W. R., and Spiro, H. M. (1963). Esophageal motility in infants and children. Pediatrics, 31, 382. 